Interfaces and Free Boundaries 14 (2012), 153-165

DOI 10.4171/IFB/277

\title{
Density estimates for phase transitions with a trace
}

\author{
YANNICK SIRE \\ Université Aix-Marseille 3, Paul Cézanne - LATP Marseille, France \\ E-mail: sire@cmi.univ-mrs.fr \\ ENRICO VALDINOCI \\ Università di Milano, Dipartimento di Matematica, 20133 Milano, Italy \\ E-mail: enrico@mat.uniroma3.it
}

[Received 20 December 2010 and in revised form 22 February 2012]

\begin{abstract}
We consider a functional obtained by adding a trace term to the Allen-Cahn phase segregation model and we prove some density estimates for the level sets of the interfaces.

We treat in a unified way also the cases of possible degeneracy and singularity of the ellipticity of the model and the quasiminimal case.

2010 Mathematics Subject Classification: Primary 35J20, 35J70, 35J75, 82B26

Keywords: Singular and degenerate boundary reaction equations, measure theoretic estimates of the level lets of phase transition layers.
\end{abstract}

\section{Introduction}

Let $p \in(1,+\infty), n \geqslant 2$ and $\Omega$ be an open bounded subset of $\mathbb{R}^{n}$. For any $u \in W_{\mathrm{loc}}^{1, p}\left(\mathbb{R}^{n},[-1,1]\right)$, we define the functional

$$
\varepsilon_{\Omega}(u):=\int_{\Omega \cap \mathbb{R}_{+}^{n}}|\nabla u(x)|^{p}+F(u(x)) d x+\int_{\Omega \cap\left\{x_{n}=0\right\}} G\left(u\left(x^{\prime}, 0\right)\right) d x^{\prime},
$$

where we used the notation $x:=\left(x^{\prime}, x_{n}\right) \in \mathbb{R}^{n-1} \times \mathbb{R}$ and $\mathbb{R}_{+}^{n}:=\mathbb{R}^{n-1} \times(0,+\infty)$. Also, for any $R>0$ and any $x \in \mathbb{R}^{n}$, we denote by $B_{R}^{n}(x)$ the Euclidean, open, $n$-dimensional ball centered at $x$, and $B_{R}^{n}:=B_{R}^{n}(0)$. We set $B_{R}^{+}(x):=B_{R}^{n}(x) \cap \mathbb{R}_{+}^{n}, B_{R}^{+}:=B_{R}^{+}(0)$, and we use the short notation

$$
\varepsilon_{R, x_{o}}(u):=\varepsilon_{B_{R}\left(x_{o}\right)}(u)=\int_{B_{R}^{+}\left(x_{o}\right)}|\nabla u(x)|^{p}+F(u(x)) d x+\int_{B_{R}^{n-1}\left(x_{o}\right)} G\left(u\left(x^{\prime}, 0\right)\right) d x^{\prime} .
$$

We will suppose that $F$ and $G$ are non-negative "double-well" potentials. More precisely, and in fact more generally, we assume that there exists $C_{o} \geqslant 1$ such that, for any $\tau \in \mathbb{R}$, we have

$$
\max \{F(\tau), G(\tau)\} \leqslant C_{o}\left(1-\tau^{2}\right)^{p} \text { and } F(\tau) \geqslant \frac{1}{C_{o}}\left(1-\tau^{2}\right)^{p} .
$$

A paradigmatic example is given by $F(\tau)=G(\tau)=\left(1-\tau^{2}\right)^{p}$, but more general potentials are allowed by (2). The gradient term in (1) is reminiscent of a $p$-Laplacian partial differential equation (hence, it encodes a possibly singular or degenerate ellipticity). We remark that the functional in (1) 
reduces to the standard Allen-Cahn phase segregation model when $G$ is identically zero and $\Omega$ lies in $\left\{x_{n}>0\right\}$. Thus, in a sense, the functional in (1) represents a phase transition in $\mathbb{R}_{+}^{n}$ with a double-well $G$ keeping track of a phase segregation on the trace of $\Omega$ along $\left\{x_{n}=0\right\}$ and it may be seen as a toy-model to understand the more complicated phenomena arising in non-local phase transitions, which have been the object of an extensive study in recent years (see, among the others, $[1-3,10]$ and also $[5,6,15,16]$ for a relation between fractional operators and boundary reactions). In practical situations, the non-local effects may be the consequence of a long-range interaction between particles, as it happens in some statistical mechanics models (see, e.g., [7]).

The trace term $\int_{\Omega \cap\left\{x_{n}=0\right\}} G\left(u\left(x^{\prime}, 0\right)\right) d x^{\prime}$ may also be considered as a model for taking into account the effect of the boundary of the container in which the phase transition occurs: in this framework, the container is $\mathbb{R}_{+}^{n}$, which, of course, up to a blow up, is a simplified, but effective, version of a smooth container when we are interested in the behavior near its boundary. In this sense, we hope that this paper may be as a first step towards a more comprehensive study of the geometric features of the phase transitions under even more severe boundary and non-local effects.

Given $Q \geqslant 1$, we say that $u$ is a $Q$-minimizer if $\varepsilon_{\Omega}(u)<+\infty$ and

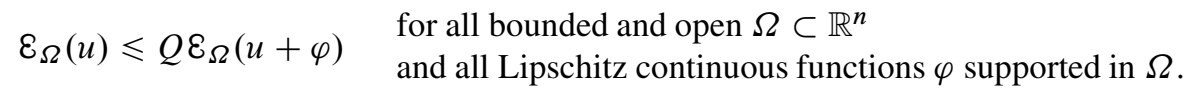

The case of $Q$-minimizers in a fixed domain $\Omega_{o}$ may be treated in a similar way (just suppose that $\Omega \subseteq \Omega_{o}$ in (3) and so on). The study of $Q$-minimizers is a classical topic in the calculus of variations (see, e.g., [9]). When $Q=1$ in (3), $u$ is usually said to be a minimizer. It is easily seen that when $p=2$ the minimizers satisfy the partial differential equation problem with Neumann condition

$$
\begin{cases}2 \Delta u=F^{\prime}(u) & \text { in } \mathbb{R}_{+}^{n}, \\ 2 \partial_{x_{n}} u=G^{\prime}(u) & \text { on }\left\{x_{n}=0\right\} .\end{cases}
$$

Such type of problems have been studied in [6,15]. Analogously, the minimizers for $p \in(1,2) \cup$ $(2,+\infty)$ satisfy a quasilinear partial differential equation whose ellipticity becomes singular or degenerate at the critical points of the solution, and the corresponding Neumann condition becomes non-linear too: these types of problem have been studied, for instance, in [16].

This is the main result of this paper:

THEOREM 1 Let $\mathcal{L}^{n}$ denote the $n$-dimensional Lebesgue measure. Let $u$ be a Lipschitz continuous $Q$-minimizer.

Then, there exists a positive $C_{*}$, only depending on $n, Q, p$, the quantity $C_{o}$ in (2) and the Lipschitz constant of $u$, such that

$$
\varepsilon_{R, x_{o}}(u) \leqslant C_{*} R^{n-1}
$$

for any $x_{o} \in \overline{\mathbb{R}_{+}^{n}}$ and any $R \geqslant 1$.

Furthermore, given any $\theta \in(-1,1)$, if we suppose that there exist two positive real numbers $\mu_{1}$ and $\mu_{2}$ such that

$$
\mathcal{L}^{n}\left(B_{\mu_{1}}^{+}\left(x_{o}\right) \cap\{u>\theta\}\right) \geqslant \mu_{2},
$$

then there exist positive $r_{0}$ and $c$, which depend only on $n, Q, p, \theta, \mu_{1}, \mu_{2}$, the quantity $C_{o}$ in (2) and the Lipschitz constant of $u$, in such a way that

$$
\mathcal{L}^{n}\left(B_{r}^{+}\left(x_{o}\right) \cap\{u>\theta\}\right) \geqslant c r^{n},
$$


for any $r \geqslant r_{0}$.

Analogously, if

$$
\mathcal{L}^{n}\left(B_{\mu_{1}}^{+}\left(x_{o}\right) \cap\{u<\theta\}\right) \geqslant \mu_{2}
$$

then

$$
\mathcal{L}^{n}\left(B_{r}^{+}\left(x_{o}\right) \cap\{u<\theta\}\right) \geqslant c r^{n},
$$

for any $r \geqslant r_{0}$.

We remark that (5) (respectively, (7)) is satisfied if $u\left(x_{o}\right)>\theta$ (respectively, $u\left(x_{o}\right)<\theta$ ): in this case, $\mu_{1}$ and $\mu_{2}$ just depend on $\left|\theta-u\left(x_{o}\right)\right|$ and on the modulus of continuity of $u$.

Our Theorem 1 fits into the line of research of density estimates for phase transition, as started in [4], to which it reduces when $G:=0$ or, basically, when we look at balls $B_{r}\left(x_{o}\right)$ that do not intersect $\left\{x_{n}=0\right\}$. Namely, the purpose of this type of researches is to try to understand how the level sets of a "good" solution $u$ behave in measure. Such level sets are physically very relevant, since they represent, roughly speaking, the separation interface of the two phases +1 and -1 in the Allen-Cahn system. Also, from these measure theoretic estimates, it is possible to deduce a locally uniform convergence of the level sets at the $\Gamma$-limit, and this information plays a crucial role in some rigidity problems (see [12, 14, 19]).

Among the many extensions of [4], we recall here the ones in [11], where the $p$-Laplacian case has been considered, [8], for quasiminima, and the recent papers [17, 18], dealing with a fully non-local case.

Estimate (6) (as well as (8)) is obviously optimal (up to the constant $c$ ), because of the trivial upper bound

$$
\mathcal{L}^{n}\left(B_{r}^{+}\left(x_{o}\right) \cap\{u>\theta\}\right) \leqslant \mathcal{L}^{n}\left(B_{r}^{+}\left(x_{o}\right)\right) \leqslant \mathcal{L}^{n}\left(B_{r}\left(x_{o}\right)\right) \sim r^{n}
$$

Estimate (4) is optimal too, as shown by the case $G:=0$, taking as $u(x)=u_{o}(\omega \cdot x)$, where $\omega \in \mathrm{S}^{n-1}$ and $u_{o}: \mathbb{R} \rightarrow \mathbb{R}$ is a minimizer of the one-dimensional Allen-Cahn functional.

As far as we know, in the framework of the functional in (1), Theorem 1 of this paper is new even in the cases $p=2$ (i.e., when the diffusion term reduces to the standard Laplacian) and $Q=1$ (i.e., for minimizers).

\section{Proof of Theorem 1}

We will denote by "const" suitable positive constants (possibly different line by line) only depending on the quantities fixed in the hypotheses of Theorem 1. First of all, we prove (4). This is done by a technique well-developed after [4]: given any $x_{o} \in \overline{\mathbb{R}_{+}^{n}}$ and any $R \geqslant 1$, we take $\beta \in C^{\infty}\left(\mathbb{R}^{n}\right)$, with $\beta(x)=-1$ for any $x \in B_{R-(1 / 2)}\left(x_{o}\right)$ and $\beta(x)=1$ for any $x \in \mathbb{R}^{n}-B_{R-(1 / 4)}\left(x_{o}\right)$, with $|\nabla \beta(x)| \leqslant 50$ for any $x \in \mathbb{R}^{n}$. Let $w(x):=\min \{u(x), \beta(x)\}$. Then, $w(x)=u(x)$ in $\mathbb{R}^{n}-$ $B_{R}\left(x_{o}\right)$, and $w=-1$ in $B_{R-(1 / 2)}\left(x_{o}\right)$. So, by (3), we obtain that

$$
\begin{aligned}
\frac{1}{Q} \varepsilon_{R, x_{o}}(u) \leqslant \varepsilon_{B_{R}\left(x_{o}\right)}(w)= & \int_{\left(B_{R}^{n}\left(x_{o}\right)-B_{R-(1 / 2)}^{n}\left(x_{o}\right)\right) \cap \mathbb{R}_{+}^{n}}|\nabla w(x)|^{p}+F(w(x)) d x \\
& +\int_{\left(B_{R}^{n}\left(x_{o}\right)-B_{R-(1 / 2)}^{n}\left(x_{o}\right)\right) \cap\left\{x_{n}=0\right\}} G\left(w\left(x^{\prime}, 0\right)\right) d x^{\prime} .
\end{aligned}
$$


Moreover, we have that $|\nabla w(x)| \leqslant|\nabla u(x)|+|\nabla \beta(x)| \leqslant$ const, and so (9) gives that

$$
\begin{aligned}
\mathcal{E}_{R, x_{o}}(u) \leqslant \operatorname{const}[ & \mathcal{L}^{n}\left(\left(B_{R}^{n}\left(x_{o}\right)-B_{R-(1 / 2)}^{n}\left(x_{o}\right)\right) \cap \mathbb{R}_{+}^{n}\right) \\
& \left.\quad+\mathcal{H}^{n-1}\left(\left(B_{R}^{n}\left(x_{o}\right)-B_{R-(1 / 2)}^{n}\left(x_{o}\right)\right) \cap\left\{x_{n}=0\right\}\right)\right] \\
\leqslant & \text { const } R^{n-1},
\end{aligned}
$$

where we denoted by $\mathcal{H}^{n-1}$ the $(n-1)$-dimensional Hausdorff measure. This proves (4).

Now, we prove (6) (the proof of (8) is the same and it will be omitted). The proof of (6) that we give here is a modification of one of the proofs performed in [8], which was inspired by [13] (other approaches, as the ones in $[4,11]$ are also possible, but they may require additional assumptions). Differently from the existing literature, here some technical complications arise in order to cope with the trace term of the functional along $\left\{x_{n}=0\right\}$. Indeed, even if such a term behaves as an $(n-1)$-dimensional correction, and therefore may look negligible, it is not completely clear that it does not dangerously interact with some "area terms" arising in the density estimates, such as the bound in (4) and the subsequent quantities in (36). For this, we will have to perform some careful computation.

First, we observe that once (6) is proved for some $\theta_{o} \in(-1,-1 / 2]$, then it is proved for all $\theta \in$ $\left[\theta_{o}, 1\right)$, because

$$
\varepsilon_{r, x_{o}}(u) \geqslant \int_{B_{r}^{+}\left(x_{o}\right)} F(u) d x \geqslant \inf _{\left[\theta_{o}, \theta\right]} F \cdot \mathcal{L}^{n}\left(B_{r}^{+}\left(x_{o}\right) \cap\left\{\theta>u>\theta_{o}\right\}\right),
$$

and if (5) holds for $\theta \in\left[\theta_{o}, 1\right)$, it holds for $\theta_{o}$ too, so using (6) for $\theta_{o}$ and (4) we obtain

$$
\begin{aligned}
\mathcal{L}^{n}\left(B_{r}^{+}\left(x_{o}\right) \cap\{u>\theta\}\right) & \geqslant \mathcal{L}^{n}\left(B_{r}^{+}\left(x_{o}\right) \cap\left\{u>\theta_{o}\right\}\right)-\mathcal{L}^{n}\left(B_{r}^{+}\left(x_{o}\right) \cap\left\{\theta>u>\theta_{o}\right\}\right) \\
& \geqslant c r^{n}-\mathrm{const} \varepsilon_{r, x_{o}}(u) \\
& \geqslant c r^{n}-\mathrm{const} r^{n-1} \geqslant \frac{c}{2} r^{n}
\end{aligned}
$$

if $r \geqslant r_{o}$ and $r_{o}$ is large enough (here the "const" may depend on the fixed $\theta_{o}$ too). This would be the proof of (6) for any $\theta \in\left[\theta_{o}, 1\right)$, up to relabeling $c$, and therefore, in what follows, we will assume, without any restriction, that

$$
\theta \in(-1,-1 / 2]
$$

Moreover, we observe that the portion of space $\mathbb{R}^{n} \cap\left\{x_{n}<0\right\}$ does not play any role in Theorem 1 , in the sense that, if we define

$$
\tilde{u}(x)=\tilde{u}\left(x^{\prime}, x_{n}\right):=\left\{\begin{array}{cl}
u\left(x^{\prime}, x_{n}\right) & \text { if } x_{n} \geqslant 0, \\
u\left(x^{\prime},-x_{n}\right) & \text { if } x_{n}<0,
\end{array}\right.
$$

we have that $\tilde{u}$ is Lipschitz, since so is $u$, that $\varepsilon_{\Omega}(\tilde{u}+\varphi)=\varepsilon_{\Omega}(u+\varphi)$ for any perturbation $\varphi$ in (3), that $\tilde{u}$ is a $Q$-minimizer and that if (6) holds for $\tilde{u}$ then it holds for $u$ as well. Consequently, we replace $u$ with $\tilde{u}$ and then we drop the superscript tilde, that is we may and do suppose that

$$
u\left(x^{\prime},-x_{n}\right)=u\left(x^{\prime}, x_{n}\right) .
$$

This symmetry property will play an important role, by allowing us to disregard some trace term in a subsequent isoperimetric inequality (that is (20) below: roughly speaking, this trick will make the 
trace term in the density estimates always be weighted by the potential, thus killing any unweighted geometric measure on $\left\{x_{n}=0\right\}$ ).

We take

$T$ to be a free parameter, that in the sequel will be chosen to be suitably large, possibly in dependence of the quantities fixed in the statement of Theorem 1 , and also in dependence of a further auxiliary parameter $\varepsilon$ that will be introduced later on, after (24).

We set

$$
S(\tau):=\min \left\{(\tau+1)^{p}, 1\right\} \text { for any } \tau \in \mathbb{R} .
$$

Also, for any $x \in \overline{\mathbb{R}_{+}^{n}}$ and any $k \in \mathbb{N}$, we let $R_{k, T}:=(k+1) T+\ln 2$, and

$$
v_{k}(x):=\left\{\begin{array}{cl}
2 e^{\left|x-x_{o}\right|-(k+1) T}-1 & \text { for any } x \in B_{R_{k, T}}\left(x_{o}\right) \cap \overline{\mathbb{R}_{+}^{n}} \\
3 & \text { for any } x \in \overline{\mathbb{R}_{+}^{n}}-B_{R_{k, T}}\left(x_{o}\right)
\end{array}\right.
$$

If $x \in \mathbb{R}^{n} \cap\left\{x_{n}<0\right\}$, we also define

$$
v_{k}\left(x^{\prime}, x_{n}\right):=v_{k}\left(x^{\prime},-x_{n}\right)
$$

By construction, $v_{k}$ is Lipschitz. Furthermore

$$
\begin{aligned}
\left|\nabla v_{k}(x)\right|^{p} & =\left(2 e^{\left|x-x_{o}\right|-(k+1) T}\right)^{p} \\
& =\left(v_{k}(x)+1\right)^{p} \\
& \leqslant \operatorname{const} S\left(v_{k}(x)\right)
\end{aligned}
$$

for any $x \in B_{R_{k, T}}^{+}\left(x_{o}\right)$, and therefore, by (13), for almost every $x \in \mathbb{R}^{n}$. Furthermore, we deduce from (2) that

$$
\max \{F(\tau), G(\tau)\} \leqslant \operatorname{const} S(\tau)
$$

for any $\tau \in[-1,1]$, and that

$$
F(\tau) \geqslant \operatorname{const}(\tau+1)^{p}=\text { const } S(\tau)
$$

when $\tau \in[-1,-1 / 2]$.

We remark that

$$
\text { if } x \in \mathbb{R}_{+}^{n} \text { and }\left|x-x_{o}\right|>(k+1) T \text {, then } v_{k}(x)>1 \geqslant u(x)
$$

and so, recalling (11) and (13), we conclude that $\left\{u>v_{k}\right\}=\left\{x \in \mathbb{R}^{n}\right.$ s.t. $\left.u(x)>v_{k}(x)\right\}$ is a bounded set. Accordingly, we can make use of (3) with $\Omega:=\left\{u>v_{k}\right\}$. This, and the use of (14) 
and (15), imply the following estimate:

$$
\begin{aligned}
\int_{\left\{u>v_{k}\right\} \cap \mathbb{R}_{+}^{n}} & |\nabla u|^{p}+F(u) d x+\int_{\left\{u>v_{k}\right\} \cap\left\{x_{n}=0\right\}} G(u) d x^{\prime} \\
& =\varepsilon_{\left\{u>v_{k}\right\}}(u) \\
& \leqslant Q \varepsilon_{\left\{u>v_{k}\right\}}\left(v_{k}\right) \\
& =Q\left[\int_{\left\{u>v_{k}\right\} \cap \mathbb{R}_{+}^{n}}\left|\nabla v_{k}\right|^{p}+F\left(v_{k}\right) d x+\int_{\left\{u>v_{k}\right\} \cap\left\{x_{n}=0\right\}} G\left(v_{k}\right) d x^{\prime}\right] \\
& \leqslant \text { const }\left[\int_{\left\{u>v_{k}\right\} \cap \mathbb{R}_{+}^{n}} S\left(v_{k}\right) d x+\int_{\left\{u>v_{k}\right\} \cap\left\{x_{n}=0\right\}} S\left(v_{k}\right) d x^{\prime}\right] .
\end{aligned}
$$

Now, we make a general observation: given any Lipschitz function $w$ on a measurable set $U \subseteq \mathbb{R}^{n}$ with image in $[-1,1]$, we have

$$
\begin{aligned}
\int_{U}|\nabla w|^{p}+F(w) d x & \geqslant \mathrm{const} \int_{U}|\nabla w|(F(w))^{(p-1) / p} d x \\
& =\mathrm{const} \int_{-1}^{1}(F(\tau))^{(p-1) / p} \mathcal{H}^{n-1}(U \cap\{w=\tau\}) d \tau,
\end{aligned}
$$

due to the Young inequality and the coarea formula.

Also, we define

$$
\begin{aligned}
m_{k}(\tau): & =\left\{x \in \mathbb{R}^{n} \text { s.t. } \tau=u(x) \geqslant v_{k}(x)\right\} \\
& =\left\{u \geqslant v_{k}\right\} \cap\{u=\tau\}
\end{aligned}
$$

and

$$
\begin{aligned}
\eta_{k}(\tau): & =\left\{x \in \mathbb{R}^{n} \text { s.t. } u(x) \geqslant v_{k}(x)=\tau\right\} \\
& =\left\{u \geqslant v_{k}\right\} \cap\left\{v_{k}=\tau\right\},
\end{aligned}
$$

and we remark that

$$
\begin{aligned}
& B_{k T}^{+}\left(x_{o}\right) \cap\{u>\theta\} \subseteq\left\{x \in B_{k T}^{+}\left(x_{o}\right) \text { s.t. } u(x)>\tau>v_{k}(x)\right\} \\
& \subseteq\left\{x \in \mathbb{R}^{n} \text { s.t. } u(x)>\tau>v_{k}(x)\right\}=\left\{u>\tau>v_{k}\right\}
\end{aligned}
$$

for any $\tau \in[(\theta-1) / 2, \theta]$.

We employ the latter formula and the isoperimetric inequality to obtain

$$
\begin{aligned}
\left(\mathcal{L}^{n}\left(B_{k T}\left(x_{o}\right) \cap\{u>\theta\}\right)\right)^{(n-1) / n} & \leqslant\left(\mathcal{L}^{n}\left(\left\{u>\tau>v_{k}\right\}\right)\right)^{(n-1) / n} \\
& \leqslant \operatorname{const}\left(\mathcal{H}^{n-1}\left(\mathfrak{M}_{k}(\tau)\right)+\mathcal{H}^{n-1}\left(\eta_{k}(\tau)\right)\right),
\end{aligned}
$$

for any $\tau \in[(\theta-1) / 2, \theta]$. 
Notice that we have in the back of our mind here the symmetry in (11) and (13), since we are willing to estimate sets in (20) in the whole of $\mathbb{R}^{n}$ instead of $\mathbb{R}_{+}^{n}$ : due to such a symmetry, this choice will be paid only by a factor 2 later on: see (22). On the contrary, without this trick we would have got also a term of the form $\mathcal{H}^{n-1}\left(B_{k T}^{n-1}\right)$ in (20), and this would have risked to be too large to be controlled by the quantities in (4) and (36).

Making use of (20) and then of (19) with $U:=\left\{u \geqslant v_{k}\right\}$ and either $w:=u$ or $w:=v_{k}$, we conclude that

$$
\begin{aligned}
&\left(\mathcal{L}^{n}\left(B_{k T}^{+}\left(x_{o}\right) \cap\{u>\theta\}\right)\right)^{(n-1) / n} \\
& \leqslant \operatorname{const} \int_{(\theta-1) / 2}^{\theta}(F(\tau))^{(p-1) / p} d \tau\left(\mathcal{L}^{n}\left(B_{k T}\left(x_{o}\right) \cap\{u>\theta\}\right)\right)^{(n-1) / n} \\
& \leqslant \operatorname{const} \int_{-1}^{1}(F(\tau))^{(p-1) / p}\left(\mathcal{H}^{n-1}\left(\mathfrak{m}_{k}(\tau)\right)+\mathcal{H}^{n-1}\left(\eta_{k}(\tau)\right)\right) d \tau \\
& \leqslant \text { const }\left(\int_{\left\{u \geqslant v_{k}\right\}}|\nabla u|^{p}+F(u) d x+\int_{\left\{u \geqslant v_{k}\right\}}\left|\nabla v_{k}\right|^{p}+F\left(v_{k}\right) d x\right) .
\end{aligned}
$$

Now, we remark that

$$
\begin{aligned}
\int_{\left\{u \geqslant v_{k}\right\}}|\nabla u|^{p} & +F(u) d x+\int_{\left\{u \geqslant v_{k}\right\}}\left|\nabla v_{k}\right|^{p}+F\left(v_{k}\right) d x \\
& =2\left[\int_{\left\{u \geqslant v_{k}\right\} \cap \mathbb{R}_{+}^{n}}|\nabla u|^{p}+F(u) d x+\int_{\left\{u \geqslant v_{k}\right\} \cap \mathbb{R}_{+}^{n}}\left|\nabla v_{k}\right|^{p}+F\left(v_{k}\right) d x\right]
\end{aligned}
$$

thanks to (11) and (13).

Therefore, exploiting (21), (22), (14), (15) and (18), we obtain

$$
\begin{aligned}
\left(\mathcal{L}^{n}\left(B_{k T}^{+}\left(x_{o}\right) \cap\{u>\theta\}\right)\right)^{(n-1) / n} & \\
& \leqslant \text { const }\left[\int_{\left\{u \geqslant v_{k}\right\} \cap \mathbb{R}_{+}^{n}}|\nabla u|^{p}+F(u) d x+\int_{\left\{u \geqslant v_{k}\right\} \cap \mathbb{R}_{+}^{n}}\left|\nabla v_{k}\right|^{p}+F\left(v_{k}\right) d x\right] \\
& \leqslant \text { const }\left[\int_{\left\{u \geqslant v_{k}\right\} \cap \mathbb{R}_{+}^{n}} S\left(v_{k}\right) d x+\int_{\left\{u \geqslant v_{k}\right\} \cap\left\{x_{n}=0\right\}} S\left(v_{k}\right) d x^{\prime}\right] .
\end{aligned}
$$

That is, recalling (17),

$$
\begin{aligned}
& \left(\mathcal{L}^{n}\left(B_{k T}^{+}\left(x_{o}\right) \cap\{u>\theta\}\right)\right)^{(n-1) / n} \\
& \leqslant \text { const }\left[\int_{\left\{u \geqslant v_{k}\right\} \cap B_{(k+1) T}^{+}\left(x_{o}\right)} S\left(v_{k}\right) d x+\int_{\left\{u \geqslant v_{k}\right\} \cap B_{(k+1) T}\left(x_{o}\right) \cap\left\{x_{n}=0\right\}} S\left(v_{k}\right) d x^{\prime}\right] .
\end{aligned}
$$


We define

$$
\begin{aligned}
& \ell_{1}=\ell_{1}(k):=\int_{B_{k T}^{+}\left(x_{o}\right)} S\left(v_{k}\right) d x, \\
& \ell_{2}=\ell_{2}(k):=\int_{\left\{u \geqslant v_{k}\right\} \cap\left(B_{(k+1) T}^{+}\left(x_{o}\right)-B_{k T}^{+}\left(x_{o}\right)\right)} S\left(v_{k}\right) d x,
\end{aligned}
$$

and

$$
\ell_{3}=\ell_{3}(k):=\int_{\left\{u \geqslant v_{k}\right\} \cap B_{(k+1) T}\left(x_{o}\right) \cap\left\{x_{n}=0\right\}} S\left(v_{k}\right) d x^{\prime} .
$$

With this notation, we see that (23) can be written as

$$
\left(\mathcal{L}^{n}\left(B_{k T}^{+}\left(x_{o}\right) \cap\{u>\theta\}\right)\right)^{(n-1) / n} \leqslant \operatorname{const}\left(\ell_{1}+\ell_{2}+\ell_{3}\right) .
$$

Now, we fix a small $\varepsilon>0$ to be taken appropriately small (in fact, at the end, this $\varepsilon$ will be fixed explicitly in Lemma 1 below) and we claim that

$$
\ell_{3} \leqslant \text { const } \varepsilon k^{n-1}+C_{\varepsilon, T} k^{n-2}
$$

for a suitable $C_{\varepsilon, T}>0$. The proof of (25) is indeed a bit long and complicated and it will be completed only below (32), after some delicate computations. To prove (25), first we notice that when $\left|x_{o, n}\right|>(k+1) T$ then $B_{(k+1) T}\left(x_{o}\right) \cap\left\{x_{n}=0\right\}=\varnothing$, so $\ell_{3}=0$ and (25) is obviously true. As a consequence, we may suppose that

$$
\left|x_{o, n}\right| \leqslant(k+1) T
$$

and so we can define

$$
\rho_{k}:=\sqrt{(k+1)^{2} T^{2}-x_{o, n}^{2}}
$$

We see that

$$
B_{(k+1) T}^{n}\left(x_{o}\right) \cap\left\{x_{n}=0\right\} \subseteq B_{\rho_{k}}^{n-1}\left(x_{o}^{\prime}\right)
$$

and therefore

$$
\begin{aligned}
\ell_{3} & \leqslant \text { const } \int_{B_{(k+1) T}^{n}\left(x_{o}\right) \cap\left\{x_{n}=0\right\}}\left(v_{k}\left(x^{\prime}, 0\right)+1\right)^{p} d x^{\prime} \\
& \leqslant \text { const } \int_{B_{\rho_{k}}^{n-1}\left(x_{o}^{\prime}\right)} e^{p\left(\sqrt{\left|x^{\prime}-x_{o}^{\prime}\right|^{2}+x_{o, n}^{2}}-(k+1) T\right)} d x^{\prime} \\
& =\text { const } e^{-p(k+1) T} \int_{0}^{\rho_{k}} r^{n-2} e^{p \sqrt{r^{2}+x_{o, n}^{2}}} d r .
\end{aligned}
$$

Now, to prove (25), we distinguish two cases: either $n \geqslant 3$ or $n=2$.

If $n \geqslant 3$, we make use of (27) to conclude that

$$
\ell_{3} \leqslant \operatorname{const} \rho_{k}^{n-3} e^{-p(k+1) T} \int_{0}^{\rho_{k}} r e^{p \sqrt{r^{2}+x_{o, n}^{2}}} d r
$$


and we perform the substitution

$$
s:=\sqrt{r^{2}+x_{o, n}^{2}} .
$$

We obtain that $s d s=r d r$, hence (28) becomes

$$
\begin{aligned}
\ell_{3} & \leqslant \operatorname{const} \rho_{k}^{n-3} e^{-p(k+1) T} \int_{\left|x_{o, n}\right|}^{(k+1) T} s e^{p s} d s \\
& \leqslant \operatorname{const} \rho_{k}^{n-3}(k+1) T e^{-p(k+1) T} \int_{\left|x_{o, n}\right|}^{(k+1) T} e^{p s} d s \\
& \leqslant \operatorname{const} \rho_{k}^{n-3}(k+1) T \\
& \leqslant \operatorname{const}((k+1) T)^{n-2} \\
& \leqslant \operatorname{const}(k T)^{n-2}
\end{aligned}
$$

This proves (25) when $n \geqslant 3$, so now we deal with the proof of (25) when $n=2$ : in this case, we claim that

$$
\int_{0}^{\rho_{k}} e^{p \sqrt{r^{2}+x_{o, n}^{2}}} d r \leqslant\left(2 \varepsilon k+\tilde{C}_{\varepsilon, T}\right) e^{p(k+1) T}
$$

for a suitable $\tilde{C}_{\varepsilon, T}>0$.

To prove (30), we distinguish two sub-cases: either $\rho_{k}<\varepsilon k$ or $\rho_{k} \geqslant \varepsilon k$.

If $\rho_{k}<\varepsilon k$, we have that

$$
\int_{0}^{\rho_{k}} e^{p \sqrt{r^{2}+x_{o, n}^{2}}} d r \leqslant e^{p \sqrt{\rho_{k}^{2}+x_{o, n}^{2}}} \rho_{k}=e^{p(k+1) T} \rho_{k} \leqslant e^{p(k+1) T} \varepsilon k,
$$

and this proves (30) in the sub-case $\rho_{k}<\varepsilon k$.

Now, we prove (30) in the sub-case $\rho_{k} \geqslant \varepsilon k$. In this case, we have that

$$
\sqrt{(\varepsilon k)^{2}+x_{o, n}^{2}} \leqslant \sqrt{\rho_{k}^{2}+x_{o, n}^{2}}=(k+1) T .
$$

Hence

$$
\int_{0}^{\varepsilon k} e^{p \sqrt{r^{2}+x_{O, n}^{2}}} d r \leqslant e^{p \sqrt{(\varepsilon k)^{2}+x_{O, n}^{2}}} \varepsilon k \leqslant e^{p(k+1) T} \varepsilon k .
$$

Now, we make the substitution in (29) to see that

$$
\begin{aligned}
\int_{\varepsilon k}^{\rho_{k}} e^{p \sqrt{r^{2}+x_{o, n}^{2}}} d r & =\int_{\sqrt{(\varepsilon k)^{2}+x_{o, n}^{2}}}^{(k+1) T} e^{p s} \frac{s}{\sqrt{s^{2}-x_{o, n}^{2}}} d s \\
& \leqslant \int_{\sqrt{(\varepsilon k)^{2}+x_{o, n}^{2}}}^{(k+1) T} e^{p s} \frac{(k+1) T}{\sqrt{\left((\varepsilon k)^{2}+x_{o, n}^{2}\right)-x_{o, n}^{2}}} d s \\
& \leqslant \frac{(k+1) T}{\varepsilon k} \int_{-\infty}^{(k+1) T} e^{p s} d s \\
& \leqslant \frac{2 T}{p \varepsilon} e^{p(k+1) T} .
\end{aligned}
$$


From this and (31), we conclude that

$$
\begin{aligned}
\int_{0}^{\rho_{k}} e^{p \sqrt{r^{2}+x_{o, n}^{2}}} d r & =\int_{0}^{\varepsilon k} e^{p \sqrt{r^{2}+x_{o, n}^{2}}} d r+\int_{\varepsilon k}^{\rho_{k}} e^{p \sqrt{r^{2}+x_{o, n}^{2}}} d r \\
& \leqslant e^{p(k+1) T}\left(\varepsilon k+\frac{2 T}{p \varepsilon}\right)
\end{aligned}
$$

This completes the proof of (30) in the sub-case $\rho_{k} \geqslant \varepsilon k$ too.

Having completed the proof of (30), we use it to complete the proof of (25) in the case $n=2$ : indeed, by (27) and (30), when $n=2$ we have

$$
\ell_{3} \leqslant \text { const } e^{-p(k+1) T} \int_{0}^{\rho_{k}} e^{p \sqrt{r^{2}+x_{o, n}^{2}}} d r \leqslant \operatorname{const}\left(\varepsilon k+\tilde{C}_{\varepsilon, T}\right)
$$

This proves (25) also in the case $n=2$. So, the proof of (25) is completed.

Now, we observe that

$$
\begin{aligned}
\ell_{1} & \leqslant \text { const } \int_{B_{k T}^{+}\left(x_{o}\right)}\left(v_{k}+1\right)^{p} d x \\
& \leqslant \operatorname{const} \int_{B_{k T}\left(x_{o}\right)} e^{p\left(\left|x-x_{o}\right|-(k+1) T\right)} d x \\
& \leqslant \operatorname{const} \int_{0}^{k T} r^{n-1} e^{p(r-(k+1) T)} d r \\
& \leqslant \operatorname{const}(k T)^{n-1} e^{-p(k+1) T} \int_{0}^{k T} e^{p r} d r \\
& \leqslant \operatorname{const}(k T)^{n-1} e^{-p T} \\
& \leqslant \varepsilon k^{n-1}
\end{aligned}
$$

provided that $T$ is sufficiently large, possibly in dependence of $\varepsilon$. This last requirement fixes $T$ once and for all (in dependence of $\varepsilon$, which, in turn, will be fixed in the forthcoming Lemma 1).

Furthermore, since $S$ is non-decreasing and bounded by 1, we obtain that

$$
\begin{aligned}
\ell_{2}= & \int_{\left\{\theta \geqslant u \geqslant v_{k}\right\} \cap\left(B_{(k+1) T}^{+}\left(x_{o}\right)-B_{k T}^{+}\left(x_{o}\right)\right)} S\left(v_{k}\right) d x \\
& +\int_{\{u>\theta\} \cap\left\{u \geqslant v_{k}\right\} \cap\left(B_{(k+1) T}^{+}\left(x_{o}\right)-B_{k T}^{+}\left(x_{o}\right)\right)} S\left(v_{k}\right) d x \\
\leqslant & \int_{\left\{\theta \geqslant u \geqslant v_{k}\right\} \cap\left(B_{(k+1) T}^{+}\left(x_{o}\right)-B_{k T}^{+}\left(x_{o}\right)\right)} S(u) d x \\
& +\mathcal{L}^{n}\left(\{u>\theta\} \cap\left(B_{(k+1) T}^{+}\left(x_{o}\right)-B_{k T}^{+}\left(x_{o}\right)\right)\right) .
\end{aligned}
$$


Moreover, using also (10), (16) and (18), and recalling (17) once more, we get

$$
\begin{aligned}
& \int_{B_{k T}^{+}\left(x_{o}\right) \cap\{u \leqslant \theta\}} S(u) d x \\
& \quad \leqslant \int_{B_{k T}^{+}\left(x_{o}\right) \cap\left\{\theta \geqslant u>v_{k}\right\}} S(u) d x+\int_{B_{k T}^{+}\left(x_{o}\right) \cap\left\{u \leqslant v_{k}\right\}} S\left(v_{k}\right) d x \\
& \quad \leqslant \operatorname{const} \int_{\left\{\theta \geqslant u>v_{k}\right\} \cap \mathbb{R}_{+}^{n}} F(u) d x+\int_{B_{k T}^{+}\left(x_{o}\right)} S\left(v_{k}\right) d x \\
& \quad \leqslant \operatorname{const}\left[\int_{\left\{u>v_{k}\right\} \cap \mathbb{R}_{+}^{n}} S\left(v_{k}\right) d x+\int_{\left\{u>v_{k}\right\} \cap\left\{x_{n}=0\right\}} S\left(v_{k}\right) d x^{\prime}+\int_{B_{k T}^{+}\left(x_{o}\right)} S\left(v_{k}\right) d x\right] \\
& \leqslant \operatorname{const}\left(\ell_{1}+\ell_{2}+\ell_{3}\right) .
\end{aligned}
$$

Now, it is convenient to introduce the following quantities:

$$
V_{r}:=\mathcal{L}^{n}\left(B_{r}^{+}\left(x_{o}\right) \cap\{u>\theta\}\right) \quad \text { and } \quad A_{r}:=\int_{B_{r}^{+}\left(x_{o}\right) \cap\{u \leqslant \theta\}} S(u) d x
$$

These quantities are appropriate variations of similar ones defined in [4], and they somewhat play the role of "volume" and "area terms", respectively, in the minimal surface analogue. By collecting the estimates in (24), (35), (33), (34) and (25), we conclude that

$$
\begin{aligned}
A_{k T}+V_{k T}^{(n-1) / n} \leqslant & \operatorname{const}\left(\ell_{1}+\ell_{2}+\ell_{3}\right) \\
\leqslant & \operatorname{const}\left[\int_{\left\{\theta \geqslant u \geqslant v_{k}\right\} \cap\left(B_{(k+1) T}^{+}\left(x_{o}\right)-B_{k T}^{+}\left(x_{o}\right)\right)} S(u) d x\right. \\
& \left.\quad+\mathcal{L}^{n}\left(\{u>\theta\} \cap\left(B_{(k+1) T}^{+}\left(x_{o}\right)-B_{k T}^{+}\left(x_{o}\right)\right)\right)+\frac{\varepsilon k^{n-1}}{2}+C_{\varepsilon, T} k^{n-2}\right] \\
\leqslant & \text { const }\left(\left(V_{(k+1) T}-V_{k T}\right)+\left(A_{(k+1) T}-A_{k T}\right)+\frac{\varepsilon k^{n-1}}{2}+C_{\varepsilon, T} k^{n-2}\right) .
\end{aligned}
$$

We define $k_{\varepsilon}$ to be the smallest integer bigger than $\mu_{1}+\left(2 C_{\varepsilon, T} / \varepsilon\right)$, where $\mu_{1}$ is as in (5). This gives that $C_{\varepsilon, T} k^{n-2} \leqslant \varepsilon k^{n-1} / 2$ and so

$$
A_{k T}+V_{k T}^{(n-1) / n} \leqslant \text { const }\left(\left(V_{(k+1) T}-V_{k T}\right)+\left(A_{(k+1) T}-A_{k T}\right)+\varepsilon k^{n-1}\right)
$$

for any $k \in \mathbb{N}$, with $k \geqslant k_{\varepsilon}$. Notice that, since $T$ has been fixed in dependence of $\varepsilon$ after (33), it is conceivable to keep track of the dependence of $k_{\varepsilon}$ on $\varepsilon$ only and disregard the dependence on $T$.

So, it is convenient to recall the following general recursive result, which is a variation of an argument in [4] and whose detailed proof may be found in Lemma 12 of [8]:

LEMMA 1 Let $C \geqslant 1, \varepsilon>0$. Let $Q_{k}$ and $V_{k}$ be two sequences of non-negative real numbers, for $k \in \mathbb{N}$.

Suppose that

$$
v_{k} \geqslant 1 / C
$$

and

$$
V_{k}^{(n-1) / n}+Q_{k} \leqslant C\left(\left(\bigcup_{k+1}-V_{k}\right)+\left(Q_{k+1}-Q_{k}\right)+\varepsilon k^{n-1}\right)
$$


for any $k \in \mathbb{N}$.

Let

$$
c:=\min \left\{\frac{1}{C}, \frac{1}{(2 C(n+1) !)^{n}}\right\} .
$$

Suppose that

$$
\varepsilon \leqslant \min \left\{\frac{c}{4 C}, \frac{c^{(n-1) / n}(\sqrt[n]{2}-1)}{2 C}\right\} .
$$

Then,

$$
Q_{k}+v_{k} \geqslant c k^{n}
$$

for any $k \geqslant 4 C(n+1)$ !.

With this, we define $Q_{k}:=A_{\left(k+k_{\varepsilon}\right) T}$ and $V_{k}:=V_{\left(k+k_{\varepsilon}\right) T}$, we have that $V_{k} \geqslant V_{\mu_{1}} \geqslant \mu_{2}$, by (5), and so (38) holds true, if $C$ is chosen large enough. Also, (39) follows from (37), again by choosing $C$ appropriately large.

Hence, we can exploit Lemma 1 (notice that (40) fixes now the value of $\varepsilon$ ), and we deduce from (41) that

$$
A_{k T}+V_{k T} \geqslant \text { const } T^{n} k^{n}
$$

as long as $k$ is large enough.

Since, by (10), (16) and (4), we have that

$$
A_{r} \leqslant \text { const } \int_{B_{r} \cap\{u \leqslant \theta\}} F(u) d x \leqslant \text { const } r^{n-1}
$$

for any $r \geqslant 1$, we conclude that $V_{r} \geqslant$ const $r^{n}$ for any $r$ suitably large, that is (6).

Acknowledgements. Both authors are supported by the ERC project $\varepsilon$ "Elliptic Pde's and Symmetry of Interfaces and Layers for Odd Nonlinearities". EV is supported by the FIRB project "Analysis and Beyond" and the PRIN project "Nonlinear Elliptic problems in the study of vortices and related topics". Part of this work was carried out while YS was visiting the Università di Roma Tor Vergata.

\section{REFERENCES}

1. Alberti, G. \& Bellettini, G., A non-local anisotropic model for phase transitions: asymptotic behaviour of rescaled energies, European J. Appl. Math. 9 (1998), 261-284. Zb10932.49018 MR1634336

2. Alberti, G., Bouchitté, G. \& Seppecher, P. Un résultat de perturbations singulières avec la norme $H^{1 / 2}$, C. R. Acad. Sci. Paris Sér. I Math. 319 (1994), 333-338. Zb10845. 49008 MR1289307

3. Alberti, G., Bouchitté, G. \& Seppecher, P., Phase transition with the line-tension effect, Arch. Rational Mech. Anal. 144 (1998), 1-46. Zbl0915. 76093 MR1657316

4. Caffarelli, L. A. \& CóRdoba, A., Uniform convergence of a singular perturbation problem, Comm. Pure Appl. Math. 48 (1995), 1-12. Zb10829.49013 MR1310848

5. CABRÉ, X. \& CINTI, E., Energy estimates and 1-D symmetry for nonlinear equations involving the half-Laplacian, Discrete Contin. Dyn. Syst. 28 (2010), 1179-1206. Zbl1193. 35242 MR2644786 
6. Cabré, X. \& SolÀ-Morales, J., Layer solutions in a half-space for boundary reactions, Comm. Pure Appl. Math. 58 (2005), 1678-1732. Zbl1102.35034 MR2177165

7. DiRr, N. \& ORLANDI, E., In preparation (2010).

8. Farina, A. \& VAldinoci, E., Geometry of quasiminimal phase transitions, Calc. Var. Partial Differential Equations 33 (2008), 1-35. Zb11156. 35018 MR2413100

9. Giaquinta, M. \& Giusti, E., Quasiminima, Ann. Inst. H. Poincaré Anal. Non Linéaire 1 (1984), 79107. MR0778969

10. Del MAR GonzÁlez, M., Gamma convergence of an energy functional related to the fractional Laplacian, Calc. Var. Partial Differential Equations 36 (2009), 173-210. Zbl1175. 49013 MR2546026

11. Petrosyan, A. \& Valdinoci, E., Density estimates for a degenerate/singular phase-transition model, SIAM J. Math. Anal. 36 (2005), 1057-1079 (electronic). Zbl1162. 35381 MR2139200

12. SAVIn, V.-O., Phase transitions: Regularity of flat level sets, ProQuest LLC, Ann Arbor, MI, 2003, Thesis (Ph.D.) - The University of Texas at Austin. MR2705495

13. SAVIN, O., Personal communication, 2007.

14. Savin, O., Regularity of flat level sets in phase transitions, Ann. of Math. (2) 169 (2009), 41-78. Zbl1180.35499 MR2480601

15. SiRE, Y. \& VALDINOCI, E., Fractional Laplacian phase transitions and boundary reactions: a geometric inequality and a symmetry result, J. Funct. Anal. 256 (2009), 1842-1864. Zbl1163. 35019 MR2498561

16. SiRe, Y. \& VAldinoci, E., Rigidity results for some boundary quasilinear phase transitions, Comm. Partial Differential Equations 34 (2009), 765-784. Zbl1188.35091 MR2560300

17. Savin, O. \& VAldinoci, E., Density estimates for a variational model driven by the Gagliardo norm, Preprint (2010).

18. Savin, O. \& VAldinoci, E., Density estimates for a nonlocal variational model via the Sobolev Inequality, SIAM J. Math. Anal. 43 (2011), 2675-2687. Zbl1233. 35201 MR2873236

19. VAldinOCI, E., SCIUnZI, B. \& SAVIN, V.-O., Flat level set regularity of $p$-Laplace phase transitions, Mem. Amer. Math. Soc. 182 (2006). Zbl1138.35029 MR2228294 